Pedagogía y Saberes n. ${ }^{\circ} 54$ Universidad Pedagógica Nacional

Facultad de Educación. 2021. pp. 97-109

\title{
El linaje técnico de la Didáctica Magna*
}

\section{The Technical Lineage of the Great Didactic A linhagem técnica da Didáctica Magna}

Aliex Trujillo**

\section{Para citar este artículo}

Trujillo, A. (2021). El linaje técnico de la Didáctica Magna. Pedagogía y Saberes, (54). https:// doi.org/10.17227/pys.num54-11807

* El artículo es derivado de un proyecto de investigación financiado por el Centro de Investigación de la Universidad Pedagógica Nacional (CIUP), bajo el título: "El campo y la pedagogía. Un análisis a propósito de la Didáctica magna de Comenio". Dicha investigación está a cargo de los grupos de investigación adscritos a la Maestría en Educación: Filosofía y enseñanza de la filosofía y Educación, pedagogía y subjetividades.

** Profesor de la Universidad Pedagógica Nacional. Correo: atrujillog@pedagogica.edu.co 


\section{Resumen}

Los saltos en los esquemas técnicos van de la abstracción magnífica en la artificialidad, hasta la concretización en lo humano. El esquema técnico del preceptor privado medieval tiene un salto y da paso a uno técnico del preceptor público, así es como aparece en la obra Didáctica Magna de Jan Amos Comenius. La educación, con la didáctica, se vuelve sistema, se concretiza con un salto en los esquemas de la técnica educativa y se acopla como cobertura de un servicio de conocimiento. El artículo es una reflexión dentro de un proyecto financiado por el Centro de Investigación de la Universidad Pedagógica Nacional (CiuP), bajo el título: "El campo y la pedagogía. Un análisis a propósito de la Didáctica magna de Comenio".

\section{Palabras clave}

analogía (operación); cobertura educativa; didáctica; técnica

\section{Abstract}

The leap in the technical schemes of the magnificent abstraction in artificiality, to the concretization in the human. The technical scheme of the medieval private tutor has a jump (leaps) in its technical schemes, it gives way to the technical scheme of the public tutor, this is how it appears in Jan Amos Comenius' Didactic Magna. Education, with didactics, becomes a system, is concretized with a jump in the schemes of educational technique and is coupled as a coverage of a knowledge service. The article is a reflection within a project financed by the Research Centre of the National Pedagogical University (CIUP), under the title: "The field and pedagogy. An analysis of the great Didactics of the Comenius".

\section{Keywords}

analogy (operation); coverage of education; didactic; technique

\section{Resumo}

Os saltos nos esquemas técnicos vão da abstração magnífica na artificialidade, até a concretização no humano. 0 esquema técnico do preceptor privado medieval tem um salto em seu esquema técnico, para o esquema técnico do preceptor público, tal como aparece na obra Didática Magna de Jan Amos Comenius. A educação, com a didática, vira sistema, se concretiza com um salto nos esquemas da técnica educativa e se acopla como cobertura de um serviço de conhecimento. 0 artigo é uma reflexão no âmbito de um projecto financiado pelo Centro de Investigação da Universidade Pedagógica Nacional (CIUP), sob o título: “0 campo e a pedagogia". Uma análise da grande Didáctica do Comenius.

\section{Palavras-chave}


Deben examinarse todas las partes del objeto, aun las más insignificantes, sin omitir ninguna; con expresión del orden, lugar y enlace que tienen unas con otras.

Jan Amos Comenius, Didáctica Magna, 2013, [XX, 20]

\section{Presentación}

En la introducción que le hace Mariano Fernández Enguita a la edición Akal de Didáctica Magna, el sociólogo de la educación dice,

Basta echar un vistazo a la historia de la pedagogía para ver que una empresa de la dimensión de la Didáctica Magna, semejante intento de pensar sistemáticamente la educación y sus técnicas, carecía de precedentes y tardó en encontrar una continuación. (Comenius, 2013, p. 24)

De acuerdo con estas consideraciones editoriales, en el presente artículo, la didáctica es un objeto técnico con cierto linaje que, "es la base y la estabilidad del esquema concreto de invención organizadora en los desarrollos sucesivos lo que funda la unidad y la distinción de un linaje técnico" (Simondon, 2007, p. 67).

La obra de Jan Amos Comenius, Didáctica Magna es, entre otras muchas cosas, un temprano manual técnico para enseñar, al mismo tiempo, a una mayor cantidad de niños y jóvenes en "escuelas en las que se enseñe todo a todos y totalmente" (Comenius, 2013, [XI, 1]). Los apartados que se toman de la obra son los necesarios para describir unos esquemas técnicos de los objetos educativos como la didáctica, en tanto esquemas que han mostrado un tipo de efectividad desde la circulación de la Didáctica Magna.

¿Cuál es el lugar que ocupa esta didáctica en un linaje técnico, operando con una analogía donde la didáctica es un objeto técnico? La didáctica y el objeto técnico se relacionan en una analogía, y la formalización de dicha relación ofrece la posibilidad de ensayar dos posibles saltos de los esquemas en el objeto técnico. Un salto sistémico con la aparición de la obra de Comenius y otro posible salto, en una fórmula que no puede ahorrar el esfuerzo del que aprende. El salto sistémico que opera en una obra del siglo XVII (que ya se detallará), y el salto posible que opera diversificando la disposición de un legado de la cultura, centrado en la relación con el saber, dispuesta una asimetría y trabajando en el deseo de saber, que es un proceso incierto, más que un conocimiento. Siendo este último, el conocimiento, el que puede diseminarse como cobertura y, por lo tanto, administrarse. Por otro lado, la didáctica en el saber, no en el conocimiento, supone un lazo social, la mediación de la condición humana. Tanto un manual como una plataforma digital tienen la mediación de un aparato que no toca la condición humana, por cuanto tienen razón de operación, pueden repetir y depurar.

El artículo abre un campo de discusión a partir de los conceptos de linaje y objeto técnicos desde Gilbert Simondon. Así las cosas, se evalúa las posibilidades de la didáctica bajo estos conceptos.

\section{Esquema técnico en la Didáctica Magna: imitar, valorar y enmendar}

La estructura de contenidos en la diagramación de la traducción al español de Didáctica Magna tiene un esquema que se repite varias veces. La traducción de Saturnino López Peces divide, en una estructura de títulos semejante al recetario, porque producen una categorización que debiera facilitar la lectura, tal como pretende el contenido de esta gran didáctica. Para facilitar la recepción por parte de los hispanoparlantes de la Didáctica Magna, se destaca la separación ficticia de los apartados. En la recepción moderna de esta obra es importante que la dificultad de su lectura sea acompañada de una herramienta como la separación temática, para mejorar la comprensión. Esto es lo mismo que recomienda la didáctica que se lee en Comenius.

En dicha estructura artificial de la traducción hay tres títulos que se repiten: un error en la imitación de la naturaleza o las profesiones, susceptible de ser enmendado, por ejemplo, con los siguientes consejos:

Toda arte debe ser encerrada en reglas brevísimas, pero muy exactas. Toda regla ha de ser expresada en muy pocas palabras, pero claras en extremo. A toda regla han de acompañarse muchos ejemplos para que su utilidad sea manifiesta, por muchas aplicaciones que la regla tenga. (Comenius, 2013, [XVII, 24])

La división temática en la traducción es una estructura con el espíritu de la Didáctica Magna, o sea, imitación (reflejo), error (evaluar) y enmienda. La imitación es la semejanza con los animales, las plantas y las profesiones. Seguir el ejemplo de estas formas que han tenido su éxito es la marcada lección del pedagogo moravo. La educación de los nuevos está plagada de errores, dice Comenius y diseña un objeto técnico (un sistema educativo) que compite con la eficacia de un ave instintiva que alimenta y protege o la eficacia de un arquitecto entrenado en el oficio de la edificación. Le sigue la enmienda, educar como hacen su labor los animales, las semillas o los oficios, porque tienen resultados tomados como aceptables 
por una cultura. Comenius, en su semejanza, destaca en el ave aquello que ha obrado sobre ella, mientras que en el arquitecto destaca la forma en que él mismo obra sobre el mundo. Según Simondon habría una diferencia entre semejanza y analogía, "la semejanza que sería únicamente una relación de identidad, en general parcial" y "La analogía es el fundamento de la posibilidad de pasaje de un término a otro sin negación de un término por el siguiente" (Simondon, 2007, p. 207). La Didáctica Magna, como objeto técnico, adopta el modelo de la semejanza.

Se deduce claramente de lo dicho que la condición del hombre y la de la planta son semejantes. Pues, así como a un árbol frutal (manzano, peral, higuera, vid) puede desarrollarse por sí mismo, pero silvestre y dando frutos silvestres también; es necesario que si ha de dar frutos agradables y dulces sea plantado, regado y podado por un experto agricultor. (Comenius, 2013, [VII, 1])

La educación debe ser como han sido domesticadas las plantas y como edifican las artes de los oficios, como crea el hombre. Si Comenius está de acuerdo con lo que hacen los arquitectos, entonces estos fueron bien educados, por lo tanto ¿por qué reformar la educación si está cumpliendo su propósito, educar arquitectos que hacen su trabajo correctamente? La cartilla didáctica no es para los arquitectos, es para aquellos que tendrán una idea de lo que hace un arquitecto, he ahí una recontextualización, tal como se expondrá más adelante. Estudiar cómo les ha dado resultado a otros, esa es la propuesta de Comenius, para que esto tenga efectos semejantes, no análogos.

El ejemplo del arquitecto (Comenius, 2013, [XVI, 5; XVI, 39; XVI , 58; XVI, 21; XVII, 4; XVII, 7; XVII, 14; XVII, 31 ; XVIII, 42]) demuestra que la forma en que hacen las cosas los arquitectos es modelo para enmendar los errores en la educación familiar, "si tratan a los discípulos con amor, fácilmente robarán su corazón de tal modo que prefieran estar en la escuela mejor que en su casa" (Comenius, 2013, [XVII, 16]). El arquitecto es educado para que edifique con éxito, que es la confirmación de las bondades de un tipo de educación. No es esa educación la que quiere Comenius, porque no se puede mantener el torrente intemporal de la cultura si todos beben de él. La educación del arquitecto no es el modelo directo de Comenius, es el proceder que resulta de este modelo. Recontextualizar el proceder de los oficios y las disciplinas es una técnica que se le atribuye a la didáctica, facilitar, seleccionar, disciplinar, homogeneizar y regular.

\section{Analogía con la didáctica}

En cambio, la didáctica es análoga a un objeto técnico (no semejante), por lo tanto, con un lugar en un linaje técnico, lugar de los esquemas de operaciones técnicas. La operación de analogía, a su vez, es otro objeto técnico de interpolación entre conjuntos, dada la proliferación inevitable de objetos que van sucediendo en un elemento, individuo o conjunto técnico.

la analogía se sostiene por lo que se podría denominar la operación fundamental de existencia de los seres, lo que hace que exista en ellos un devenir que los desarrolla haciendo aparecer figura y fondo; el pensamiento técnico solo capta las estructuras figurales de los seres, que asimila a sus esquemas.

(Simondon, 2007, p. 207)

En la analogía dos conjuntos diferentes se relacionan "incompatibles, pero no opuestos" (Combes, 2017 p.38). Se tienen estos dos conjuntos, el conjunto $A$ y el conjunto $B$ se diferencian en las propiedades de los elementos que convocan respectivamente. Sea $a 1$ un elemento que pertenece a $A$ y que tiene otra propiedad en potencia: $C$. De igual forma $b 1$ tiene una propiedad en potencia, también $C$. Entonces, el elemento $a 1$ y $b 1$ se relacionan en un tercer conjunto $C$, que actualiza la propiedad $C$ que está en potencia. Este es el esquema de la analogía, una operación que produce relaciones entre elementos compartidos y en potencia, una potencia activada en $C$. Supongamos $A$ los elementos rojos, $B$ los elementos amarillos. En $A$ hay autos rojos y medias de ese color. En $B$ hay autos amarillos, empanadas, amapolas, un vestido, etcétera. El auto amarillo y el auto rojo hacen conjunto, el conjunto de los autos. El conjunto de los autos es una analogía.

Con la didáctica una analogía sería: el linaje del objeto técnico como la didáctica va de un estado de abstracción a la concretización. Lo abstracto es la operación con la que se le arranca al mundo la funcionalidad de un objeto, es la artificialidad que lo separa de este, la forma radical de un salto inicial. El salto es una transformación operativa de los esquemas técnicos que tienden a esquemas concretizados. Como la physis, o el mundo natural, el salto está cargado de determinaciones, de concreciones (ser de lo concreto); concretización le llama Simondon (ser de las concreciones sucesivas) y hay un salto entre una abstracción y una concretización en los esquemas de un objeto técnico. Entendiendo que "El objeto técnico no debe ser tratado como un esclavo o aprehendido como medio de juego: debe ser captado en su interioridad dinámica, en el esquematismo concreto, pero abierto, de su estructura y de su fun- 
cionamiento" (Simondon, 2017, p. 246). Desde aquí a un objeto técnico le es conferida la propiedad de tener varios esquemas técnicos, es posible que uno de estos esquemas sea aquel donde la formación en máquina (Trujillo, 2017), toma cierta didáctica como objeto técnico en estructura y funcionamiento. La concreción o abstracción de los esquemas técnicos tiene lugar en una escala que va desde la abstracción inicial de la experiencia sensible y mudable, pasando por una concreción relativa y sistémica, hasta una concreción relativa a un lazo social en las relaciones de saber.

Una posible abstracción del objeto técnico llamado didáctica consiste en arrancar del mundo el recuerdo, acuerdo y conflicto de un conjunto de producciones a conservar, para entregar como proceso al que no lo solicita. En cambio, la concretización de unas operaciones didácticas podría apuntar a la disposición amplia de las formas de unas conquistas del saber. Toda didáctica tiene que enfrentarse a la contingencia de lo humano, donde habita el deseo de saber que no es congénito ni es la única opción. Por lo tanto, pertenece a la dificultad en el acceso a la cultura, en el que hay que trabajar desde que no se quiere hasta que se quiera. Aquí hay una energía atravesada, un trabajo, una fuerza con resultados de una índole minuciosa. Lo imposible es anticipar los resultados. El nuevo en el mundo no puede solicitar aquello que aún no desea. Así, una queja de los padres es este muchacho ni sabe lo que quiere. Esta queja es elocuente, el nuevo no tiene por qué saber lo que quiere, o puede no querer, o no puede querer saber. Ni siquiera se trata de saber o no, es posible que el problema esté en que ese querer hay que formarlo y la formación es transindividual (Simondon, 2009, p. 371). Lo transindividual para Simondon es la emoción, afección, percepción y acción, en la producción del lugar que hace ser a un sujeto, por estar sujeto, en este caso de la educación, al saber. El medio asociado "potencias, en el sentido más amplio del término, es decir, capacidades de producir o de padecer un efecto de una manera determinada" (Simondon, 2007, p. 94), a un objeto técnico, como cierta didáctica, es la cobertura. Esta didáctica y la cobertura tienen una relación de mutuo afecto (trasindividual); donde el contenido, como conocimiento, produce una administración que puede (o no) tener como efecto un saber. Este esquema técnico, la relación entre didáctica y cobertura, no ha cambiado desde la Didáctica Magna.

La didáctica y el objeto técnico, análogos por su esquema técnico producen la administración de la educación pública que desde Comenius no deja de ser la misma, un ser de operaciones que toma el reloj como semejanza procedimental. Estas operaciones de la didáctica tienen una serie de problemas de orden técnico:

\begin{abstract}
¿Cómo un solo Preceptor puede ser suficiente para cualquier número de discípulos? [...] ¿Cómo puede ser que con unos mismos libros se instruyan todos? [...] ¿Cómo puede hacerse que a un mismo tiempo todos hagan lo mismo en la escuela? [...] ¿Cómo puede hacerse que para todo se emplee el mismo método? [...] ¿Cómo puede llenarse el entendimiento de muchas cosas con muy pocas palabras? [...] ¿Cómo deben enseñarse las cosas para obtener doble o triple resultado con un solo trabajo? [...] ¿Cómo podrå hacerse todo gradualmente? (Comenius, 2013, [XIX, 16, 31, 38, 40, 41, 42, 51, 52])
\end{abstract}

Desde Comenius, la educación con sus metas y su didáctica con sus propósitos pedagógicos opera distinto a la formación, entendida esta como uno de los posibles efectos de una estructura educativa. Frente a esto, una posibilidad es que para la formación haya que renunciar a toda clase de didáctica, porque la formación es imposible de anticipar. La abstracción de la didáctica está relacionada con pretender controlar el propósito, una y otra vez; la concretización, grado que se considerada opuesto a la abstracción, fue posible hasta ahora con la didáctica de Comenius y esto ha sido un salto en el esquema técnico. Dicho salto puede estar relacionado con la pluralidad y profundidad del legado cultural, por principio, puesto a considerar en la escuela. Para la formación se pueden enriquecer las situaciones donde los nuevos cuenten con todos los recursos intelectuales de la humanidad que puedan respaldar y asegurar sus elecciones.

Es posible que la didáctica produzca una formación - que no pudiendo anticiparse- tome un movimiento imprevisto hacia el deseo de saber, en la dirección del saber como posibilidad. A pesar de la didáctica habría la posibilidad, la que tiene una dificultad cualquiera, en tanto ser el objeto de la dificultad. La dificultad con el saber es el proceso de una didáctica que dispone la instrucción que dificulta, como condición de posibilidad del conocimiento de sí, operación que pone la cultura a disposición del deseo de saber, que es un efecto que ocurre después de ingresar a la cultura. Se podría valorar la posibilidad de una didáctica en la formación, en tanto objeto técnico concretizado. Exhibir los vínculos problemáticos con el saber podría ser una recomendación, una consigna, una sentencia, el principio de una didáctica con otros esquemas técnicos que son efecto de un salto en el linaje. Sin embargo, una didáctica como la de Comenius está buscando apurar los vínculos 
con consejos dosificados sobre la planeación en un sistema de educación y que, por eso mismo, está en crisis permanente. La relación con el saber es un tipo de vínculo social que responde a principios estructurales y estos son tales porque prescinden del contexto, al no poder dejar de realizarse.

\section{Salto de saber de los esquemas técnicos}

La relación entre la formación y la didáctica puede hacerse en tres operaciones, al menos: la imposibilidad, el enriquecimiento y la indiferencia. La imposibilidad viene dada porque en la formación ocurre todo aquello del saber que no puede anticiparse, siendo este el propósito técnico de la didáctica. Si hay relación de enriquecimiento es porque se considera que enriquecer el acceso cultural requeriría de cierta didáctica o este enriquecimiento sería ya una didáctica. En la operación de indiferencia, los contenidos y movimientos de la didáctica, en un esquema técnico, pueden ser cualquiera para la formación.

Para incrementar la participación en una selección de la cultura se usa la didáctica, donde se enseña a tomar un atajo en las posibilidades con el conocimiento y este enseñar es técnico. Con la técnica en la educación, algo de la cultura ha ido siendo cada vez más eficaz, en cada generación, ahorrando empezar siempre por la misma recolección de la piedra. El libro de texto que inaugura Comenius es más que esto, es el objeto técnico de la escritura y la lectura reproducida con productividad, el conductor de la diseminación efectiva del pastor de almas. Hay un conocimiento que se le puede enseñar a todos, sin importar quién, basta que el objeto técnico sea instrumento de la palabra (Bernstein, 1990). En Comenius, la abstracción de su didáctica es esa separación artificial del saber, es la invención del conocimiento y de ahí proviene el salto técnico.

Podría producirse un lazo social en la operación didáctica, una didáctica retroactiva que tiene dos problemas adicionales, que tendría que ser una administración de lo singular y hacer de lo contingente una necesidad. Lo singular es irreproducible, no extrapolable, imposible de diseminar y de organizar como otros, que serían del orden de lo particular. Si la didáctica es una generalización de la operación técnica de un sistema educativo, no podría haber didáctica en la formación. Que sea imposible la relación entre didáctica y formación, no quiere decir que se tenga que dejar de buscar dicha relación. Entonces, la concretización de la didáctica podría ser un salto a una integración mayor con el mundo, en la exposición a toda suerte de posibles experiencias con el saber, para un posible efecto. Este lazo puede actualizarse a condición de que el otro (el maestro) lo exhiba con entusiasmo, "cuanto mayor sea el entusiasmo mayor será la actividad que despierte en sus discípulos" (Comenius, 2013, [XIX. 16]).

En un encuentro social, a propósito del saber, el nuevo y el adulto pueden encontrar un tipo de vínculo. El adulto (maestro), exige un acceso al legado por descubrir, dispone los problemas en el saber, con los que lidia él mismo, pero el otro todavía no es un par. Precisamente, a condición de que haya esta especie de disparidad, es que se moviliza el posible vínculo con el saber, querer lo que supera al otro, estar y saberse en déficit. "Cualquier relación pedagógica es asimétrica, intrínsecamente asimétricas, porque si no es asimétrica, entonces, ¿ en qué sentido podemos hablar de que es una relación pedagógica?" (Bernstein, 2000, p. 38). El sociólogo de la educación pone una condición para que la relación sea pedagógica, que se cumpla una asimetría necesaria. Lo cual implica que, si se erradica la asimetría del saber entre el que enseña y el aprende, entonces la relación es alguna otra, menos pedagógica.

Jan Amos Comenius propone que la relación educativa pueda actualizarse por fuera de la familia, que un solo adulto le imparta, los mismos contenidos seleccionados y organizados, a muchos otros (que no lo tienen) a la vez, "un solo Preceptor sea bastante para instruir a cien alumnos con diez veces menos trabajo que el que actualmente emplean con un solo" (Comenius, 2013, [XII, 2]. En un salto técnico, los esquemas técnicos de la institutriz y el preceptor o tutor, forma otra rama del linaje técnico, en los esquemas técnicos del maestro o profesor. Desde ese salto, la didáctica ha tenido escasas transformaciones. Todavía la escuela, de cualquier nivel, es la operación de un objeto técnico muy parecido al que planteó Comenius; esto es, una operación que se presenta de lo sencillo a lo complejo, de lo general a lo particular (de la unidad a las partes) y de lo próximo a lo remoto. Estos pudieran ser esquemas técnicos que se reproducen, de la escritura y lectura de la letra, a las redacciones; de los principios de la gramática, a la retórica y dialéctica; y del contexto al universo, de los números a las operaciones, de las piezas a la máquina, del reloj al cosmos. En este sentido es declarado por Comenius el esquema técnico: sentidos, memoria, entendimiento y juicio

[...] porque la ciencia empieza por el sentido, y por la imaginación pasa a la memoria; después, por inducción de lo singular, se forma el entendimiento de lo 
universal, y por último, de las cosas suficientemente entendidas se compone el juicio para la certeza del conocimiento. (Comenius, 2013, [VII, 28])

La didáctica es un objeto técnico porque a pesar de la historia mantiene un esquema de universalización de lo particular, este objeto tiene una técnica que actúa con esquemas de un lugar en el linaje. Como se ha dicho, el linaje progresa de lo abstracto a lo concreto, mientras que la didáctica va de lo concreto a lo abstracto, de lo simple a lo complejo. Desde la Didáctica Magna, la didáctica es un objeto técnico que empieza a organizar la educación en un sistema para la población de un estado nación, o sea, para todos. Como son todos, como todos son muchos, también propone un sistema de enseñanza donde un adulto (preceptor) pueda enseñar a muchos al mismo tiempo. La traducción al español propone una estructura para el traductor enseñar a todos, y a muchos, al mismo tiempo: imitación, error y enmienda. El traductor sigue las enseñanzas de Comenius en la estructura que le da al texto del libro, puede ser un homenaje a su método. Los errores en la enseñanza se pueden corregir, tomando como ejemplo lo que hacen los oficios y como es la naturaleza.

\section{Linaje técnico y didáctica}

La didáctica de Comenius tiene el mismo linaje que un objeto técnico como el reloj:

Hay que confiar que se hallará una organización de las escuelas semejante a la del reloj [...] Intentemos, pues, en nombre del Altísimo, dar a las escuelas una organización que responda al modelo del reloj, ingeniosamente construido y elegante decorado. (Comenius, 2013, [XIII, 15])

El reloj del que habla Comenius en la Didáctica Magna tiene un esquema técnico semejante al reloj de John Harrison ${ }^{1}$. Los esquemas de ambos relojes tienen lugares distintos en el linaje técnico, el reloj de Comenius, con el que organiza la escuela, es un reloj que tiene existencia para desempeñarse en tierra firme, ese es su medio asociado:

Como una bóveda que no es estable más que cuando está terminada, este objeto que cumple una función de relación solo se mantiene, solo es coherente, después de que existe y porque existe; crea por sí mismo su media asociado y está realmente individualizado en él. (Simondon, 2007, p. 77)

1 Famoso relojero y carpintero inglés (1693-1776) que resolvió el problema de la latitud. Su cronómetro marino tenía la precisión necesaria para determinar la latitud terrestre en la navegación, reduciendo ostensiblemente el riesgo de accidentes navales.
En cambio, el medio asociado del reloj de Harrison es el mar. En 1714 se promulgó un decreto del Parlamento Inglés, el Decreto de la longitud, se ofrecía veinte mil libras esterlinas a quien le diera solución a la medición de la longitud geográfica para navegar de forma segura al nuevo mundo (Sobel, 2006). El reloj de Harrison, el H4, es una solución a la inestabilidad de las olas, a salvo de las oscilaciones caprichosas de las naves batidas por el océano. Incluso, el H1 es un reloj más concretizado que el reloj escolar de Comenius. Sin embargo, ambos relojes tienen en común que pertenecen a sociedades calientes (Charbonnier, 2006, p. 38) aquellas que se organizan con más extensión e intensidad, para esto consumen grandes cantidades de energía. Al navegar con más precisión era admisible navegar más rápido, y se produce la solicitud técnica de potencia y al enseñar con más precisión se pudo llegar más lejos con la enseñanza.

Según Simondon (2007), el linaje es la operación de concretización en un esquema técnico, pero antes, cuando se unen dos potencias de relacionamiento del mundo, dos elementos técnicos. La abstracción ocurre porque esa unión ya estaba en el lenguaje, ya era una condición de posibilidad. Por eso, en la palanca, por ejemplo, la abstracción inicia el linaje de todo conjunto de elementos que amplifique la fuerza aprovechando la geometría. Comenius nos recuerda una geometría aprovechada por el pensamiento técnico como modelo para el funcionamiento en la escuela: "una pequeña máquina verdaderamente ingeniosa compuesta de cilindros, poleas y cuerdas, de tal modo que unos elementos ayudasen a los otros para obtener la multiplicación de las fuerzas" (Comenius, 2013, [XIII, 8]). La Didáctica Magna adopta el modelo de las máquinas de Arquímedes: semejante a la maravilla del cosmos, la escuela funciona como en un reloj.

En la abstracción, el linaje de un hacha inicia en todo aumento de la velocidad de impacto de una trayectoria curva; al aumentar el diámetro del círculo aumenta la velocidad tangencial instantánea y aumenta el impacto al multiplicarlo por la masa. La catapulta es del linaje técnico del hacha, ambas trayectorias amplifican la velocidad final de la herramienta; también pertenecen a este linaje la pesada retroexcavadora y algunas órtesis odontológicas. Estas últimas, tienen un grado de concretización mayor, puesto que se acoplan muy bien (esa es una de sus funciones) al aparato masticador humano, que es su medio asociado. En ningún lugar del universo se encuentran, de forma apropiada, un conjunto de elementos informados para un propósito como el impacto devastador, en el hacha; o la trasformación de los materiales, en el martillo. Dos seres como el palo, que ahora es mango y piedra con agujero, que 
ahora es cabeza de impacto del hacha, son inconmensurables sin el lenguaje que lo arranca del mundo y las ensambla. Esos dos lugares, el palo-mango y la piedra-herramienta unidos en la nueva herramienta, son imposibles para cualquier otra forma y magnitud de energía.

Un posible e inicial linaje de la didáctica relaciona unas operaciones cuyo esquema cambia con una asíntota de concretización del objeto técnico en el mundo. Pero no es suficiente. Se necesita, además, reconocer que el cambio en el linaje técnico tiene una existencia por saltos. El mundo natural es la concreción misma, el estado que nunca alcanza el objeto técnico, pero que no puede dejar de perseguirlo. Dice Simondon:

\begin{abstract}
No es suficiente entonces con decir que el objeto técnico es aquello cuya génesis específica procede de lo abstracto a lo concreto; hay que precisar todavía que esta génesis se cumple por medio de perfeccionamientos esenciales, discontinuos, que hacen que el esquema interno del objeto técnico se modifique a través de saltos y no según una línea continua. (Simondon, 2007, p. 61)
\end{abstract}

El linaje de un objeto técnico como la didáctica, ocurre por los saltos en los esquemas de operación. Se podría decir que las técnicas del conocimiento de sí, recontextualizadas de los griegos en el siglo de Comenius (siglo XVII), comenzaban a separar la educación y la producción (Bernstein, 1990, p. 59), dichas técnicas de separación producen un grupo de operaciones didácticas con unos esquemas técnicos dominantes. Habría operaciones didácticas que responden a un cuidado de sí con el otro, en una condición en principio especular, superable en las relaciones de deseo, en una diferencia de saber con el otro, relación que, en la escuela, está mediada por saber. Un deseo de saber necesita un reconocimiento, a propósito de una diferencia de saber. Este reconocimiento mediado opera como en el diálogo de Platón, aquel donde Sócrates interroga a Alcibíades, "Y bien Alcibíades, sea fácil o difícil, el hecho con que siempre nos enfrentamos es este: que conociéndonos a nosotros mismo podremos conocer la manera de cuidarnos mejor, cosa que, en otro caso, desconoceremos radicalmente" (Platón, 1961, [129a]). El conocimiento de sí pasa por la pupila del otro, y no puede ocurrir sin la relación con este, de ahí procede la exhortación, Conócete a ti mismo (gnóthi seautón).

Por otro lado, en el cuento de Michael Ende, $L a$ historia interminable (1985) la Emperatriz Infantil obliga al héroe a ignorar que ella conoce el resultado de la misión que le confía. Una misión, cuyo éxito es la misión misma, siempre y cuando no conozca esto por adelantado, a condición de esto último. La misión tiene éxito porque el héroe nunca sabe lo otro que está haciendo y, además, por todo lo que tiene que bregar contra los obstáculos físicos e intelectuales surcados para ser digno de sus antiguos; el héroe cumple su misión teniendo que ir forjándola. En todo caso, la misión consiste en ir construyéndola. El asunto aquí podría ser ¿qué efecto produce la condición específica donde el propósito (la misión) sea cada cosa que se lleve a cabo, a condición de no saber y, de todas formas, querer saber? Hay un obstáculo para que el hombre se conozca a sí mismo, tiene que ignorar su destino sin que seje en desentrañarlo.

La Didáctica Magna se entroniza con las técnicas que no pasan por el conocimiento de sí, aquello que nadie puede hacer por el otro, porque no es un contenido, es un proceso. De ahí que la advertencia de Platón sobre las palabras del oráculo es desoída por el pedagogo moravo, al menos en este aspecto, recontextualizó al dios griego de las primarias estatuas clásicas, en un dios sin figura, pero sí con cogito. La cultura en el esfuerzo metodológico y con la operación técnica de la didáctica es transformada en conocimiento para lo humano, con énfasis en el "para", porque hay una especie de servidumbre en esta preposición, la servidumbre concedida a los fines a la causa final. El conocimiento aquí es aquello que puede transportarse, propagarse, discriminarse, conservarse como flujos estructurados de información, como valor de cambio. Es el mismo conocimiento de los bárbaros, según Platón en el diálogo de marras, cuyas tiranías ponen la felicidad en la fastuosidad, en el intercambio de bienes y servicios. Este salto en la didáctica como objeto técnico se esfuerza por garantizar la cobertura, cosa de bárbaros eso de que todos tengan el mismo pedazo seleccionado de cultura, tomado como un proceso de control cada vez más extenso y focalizado.

En un salto técnico distinto al objeto técnico de Comenius, está la posibilidad de una didáctica concretizada. Una posible didáctica concretizada tiene un funcionamiento a favor del aumento de las posibilidades de acceso pleno a la cultura, un acceso que contemplar, trabajar y aspirar. Teniendo lo técnico como condición de lo humano, entonces se pensaría en una técnica de disposición de las heterogeneidades específicas del saber; así, ahí podría haber posibilidades para un salto de los esquemas técnicos en la didáctica. En este sentido, la didáctica concretizada es potencia de disposición porque despliega, también, algo que permanece y que es imposible de transcribir a los propósitos de un proyecto o de conocer por adelantado, como en la anécdota de Atreyu, el personaje de La historia interminable. 
Los efectos de las, eso sí, disposiciones múltiples del saber, son una posesión imposible pero deseable porque habría que formar ese deseo. La concretización de esta posible didáctica podría estar más cerca de la condición humana y por eso tiene esquemas de saber. Cada estudiante va eligiendo, dadas las opciones, una serie de posibilidades de las cuales no tiene certezas respecto al resultado. Multiplicar las opciones en la educación no ahorra trabajo, dificulta cualquier aceleración porque habría que plegarse a las condiciones de cada opción, valorando el saldo de saber en la relación que podría formarse a propósito de un legado.

Tal es así que, la didáctica de Comenius y las modernas plataformas digitales de enseñanza no tienen diferencias como objetos técnicos, ambos buscan la cobertura, también en clave cultural y económica. La cobertura disemina un legado y la acción de diseminar es una operación técnica. Ambas, el manual pedagógico del siglo xvir y un Learning Management System (LMS, por sus siglas en inglés) ${ }^{2}$ del siglo XXI, parecieran ser análogos en no considerar que está en juego la condición del deseo de saber, con otras complejidades imposibles de administrar porque, en principio, tienen tiempos distintos: cronológico y lógico.

Una recontextualización de la técnica ocurre por imitación y corrección, en los antiguos manuales escolares y por simulación y recursividad, en los omnipresentes sistemas de gestión del aprendizaje (LMS). Ambas recontextualizaciones tienen la misma causa final, diseminar la buena nueva. La recontextualización "extrae (descoloca) un discurso de su práctica y contexto sustantivos y lo recoloca según su propio principio selectivo de reordenación y enfoque" (Bernstein, 1997, p. 189). Ninguno de estos objetos técnicos, manual escolar y LMS, son necesarios en el humano lazo social. No son necesarios para querer tener el saber que exhibe el otro, ¿qué es eso que tiene el otro, que lo exhibe con tanto apego? El nuevo podría querer tener ese apego, esa emoción que muestra el otro con el saber. "El educador está causado de cierto modo y eso se convierte en un enigma para el aprendiz, quien, en consecuencia, se vuelve hacia él para tratar de responder" (Bustamante, 2017, p.73). Ese humano lazo social tiene un estatuto que no es tocado por la cobertura (diseminación), dar menos para que alcance para todos, como si el saber dependiera exclusivamente de una transacción (operación) de conocimiento o información.

2 Se refiere al software alojado en un servidor que administra los archivos con la información de un trayecto pedagógico en el aprendizaje remoto. Dicha administración, en gran parte, es asincrónica. Lo que pretende suponer como una ventaja estratégica para la educación.

\section{Objeto técnico Abstracto o concretizado}

Como se ha dicho bajo el rótulo de Linaje técnico y didáctica, lo abstracto es una máxima y primera separación del mundo. La estabilidad de un esquema técnico abstracto se ejemplifica con el hacha de piedra: se tiene una piedra afilada de forma, en principio, accidental, que se selecciona por su forma y hace resonancia con otra forma y esto informa al conjunto sobre un funcionamiento relacional. Se arranca la piedra afilada de su inmutable existencia de substancia y se amarra al extremo de un palo. Esto es una operación que demanda saturación de posibilidades y acumulado simbólico para ampliar casi al infinito esas posibilidades, lenguaje en el que poder pensar ese algo que se le arranca al mundo y lenguaje donde obra el arrancar mismo. La liana, que une a la piedra y el palo, también es arrancada (sacada de ahí). En el mismo sentido, el palo es levantado del azar por selección (duro, rígido y transformable), y la piedra (dura, afilada y acoplable). En el lenguaje, estos objetos, la piedra, la liana y el palo se elevan a la determinación de unas uniones, de un desempeño soñado, de su efectividad verificable, o sea, puesta a experimentar. Para fabricar el hacha, la piedra, además de tener filo, es seleccionada por tener un agujero o un asiento conveniente para el palo. El palo pudo ser preparado con la misma piedra afilada. Para estas operaciones transcurrieron millones de gestos que se actualizaron una y otra vez, siempre delante de otros, habitando el lenguaje compartido. La actualización fue incrementada con la participación de más insistencia en el resultado preciso. Las piedras más duras (las que menos se parten en los reiterados ensayos) pueden afilar y abrir huecos a las otras piedras. Incluso, sacar lascas en la madera, por una primacía mecánica entre estos materiales. Así ocurre la necesaria abstracción de sacar tres pedazos del mundo, piedra, palo y liana y convertirlos entre sí, en un individuo técnico en el seno del lenguaje.

La fuerza más descomunal de las estrellas, el calor encofrado en los planetas, los meteoritos, maremotos, incendios; ninguno de estos cataclismos (disipación de altos niveles de energía en poco tiempo) es suficiente para producir la extrema complejidad de tomar un pedazo del mundo. Aún más, de forma improbable, juntarlos con otros pedazos, de otros lugares, de signos muy separados en el mundo, mundo en donde cualquier distancia es abismal porque es un mundo en la substancia de lo simbólico. Estas operaciones que provocan pequeños y constantes cambios en el universo requieren extrema abstracción y, confirman su artificialidad. Dicha artificialidad es una abstracción que se da, entre otras razones, porque la labor en la 
anticipación y la realización del mundo está dominada por el objeto, el instrumento que al arrancarse de la substancia produce un sujeto. Mientras, en una concretización, o sea, el otro extremo ontogenético, el objeto que domina se convierte en un producto en medidas y características. Los oficios se enseñaban mostrando durante muchos años el efecto de la actividad secuencial y minuciosa. El aprendiz es el testigo activo de las pequeñas variaciones de los gestos, de los materiales del cuidado de la flexibilidad en las operaciones; en cambio, después de un salto técnico, las profesiones se enseñan con un legado de conocimientos, de protocolos simbólicos recontextualizados que trabajan con sus propios esquemas internos.

El aumento en la sofisticación de las operaciones técnicas, van creando conjuntos técnicos más autónomos, más cercanos al mundo, a la concertada autonomía de las reglas simples. El gran colisionador de hadrones Large Hadron Collide (LHC, por sus siglas en inglés) es un conjunto técnico concreto porque se acerca al concreto funcionamiento del universo, pero no solo hace esto. Reproduce sus procesos más elementales, soportado por las formas en que el universo ha ido cobrando relaciones con la concreción de una imitación antigua. La inteligencia artificial que controla los procesos más sofisticado dicho LHC, aprende de los pequeños errores que la predictibilidad del modelo pasó por alto, para acelerar protones a $99.99 \%$ de c (velocidad de la luz). Ese es el hombre en su ontogénesis, un ser en la imitación minuciosa de lo concreto del mundo.

La sonda marciana Curiosity es una reproducción, cada vez más refinada, de las posibilidades cercanas al mundo que el hombre es capaz de imaginar y traer a ese mismo mundo, en el que la humanidad se ha ido enseñoreando. Estos automatismos, el LHC y el Curiosity, que para el sentido común están juntos en la cumbre de la tecnicidad, son distintos. El LHC es un conjunto técnico que es tan cercano al mundo porque el hombre es un interior del conjunto, es un imprescindible elemento técnico que coordina, repara y estructura los experimentos. En cambio, el Curiosity es un automatismo inflexible, tan fino en su auto-organización que está casi cerrado a lo impredecible, a lo fortuito. El hombre queda en el exterior del individuo técnico está conectado a un conjunto técnico, pero tiene la fabricación y programación de durar para siempre, aunque no hay un para siempre en el universo. Ahí radica su artificialidad, separado del mundo para funcionar con sus leyes.

Estos dos ejemplos confirman las posibilidades de distinguir entre unos pocos tipos de objetos técnicos. Una didáctica, como objeto técnico, tiene unos esque- mas diferenciales de operación con la cultura, unos que pretenden depender del hombre y otros que no. El grado de concreción en el linaje técnico de la didáctica, toma el automatismo como abstracción, es el caso de la sonda marciana, a prueba de humano, pero dependiendo de este. Lo corresponde a este automatismo la didáctica informacional y abstracta del protocolo remoto, por ejemplo. En cambio, el acelerador emula el mundo, funciona como lo hace el universo.

\section{Cobertura, objeto técnico y didáctica}

La analogía entre el objeto técnico y la didáctica está hecha de una operación que no ha cambiado con la historia: la cobertura, o sea, educar a todos, por conveniencia de la república. Nietzsche lo presenta en Sobre el porvenir de nuestras escuelas, donde la cobertura, vendría a ser extender, diseminar la cultura. Dice Nietzsche, en el segundo prefacio a la publicación de estas conferencias, dictadas en la Sociedad Académica de Basilea,

Dos corrientes aparentemente contrapuestas, de
acción igualmente perjudicial y concordantes en sus
resultados, predominan en la actualidad en nuestras
escuelas, que originalmente partían de bases total-
mente diferentes: por un lado, la tendencia hacia
la máxima extensión de la cultura, y, por otro lado,
la tendencia a disminuirla y debilitarla. (Nietzsche,
2010, p. 24)

Nietzsche está pensando que hay una cantidad indeterminada, pero finita, de cultura. Al expandir está cantidad, se estrecha, se debilita, se alejan los núcleos de saber entre sí y de su centro auténtico.

Si se piensa en una determinada porción de masa fresca de pan, debidamente hidratada, el rodillo ayuda a expandir una mezcla elástica determinada y constante. Esta operación se realiza para introducir aire a la masa. Con el trabajo mecánico de amasado se incrementa el ingreso del oxígeno que necesita la levadura, que son bacterias que producen $\mathrm{CO}_{2}$, para incrementar el volumen de la masa cuando después reposa. Pero antes, hay que extender y doblar, volver a extender, y así. Cuando se extiende la masa con el rodillo, aumenta su área, mientras más aumenta el área, más disminuye el espesor de la masa; esto, porque la masa es constante. Un incremento produce una disminución, son contrarias y directamente proporcionales. De nuevo, esto es porque la masa de pan es aproximadamente constante. Algo así debería pasar con la cultura, al extenderla, su espesor disminuye. Según Nietzsche, la disminución del espesor es perjudicial para la cultura que más veces ha sido 
heredada. La propuesta de Nietzsche es la esfera, la agrupación alrededor de un núcleo denso y escaso. La propuesta de Comenius, en Didáctica Magna, es el disco. La misma "masa" de la cultura auténtica se esparce, con la técnica y el instrumento, en un cilindro de poca altura. La periferia tiene una mayor distancia a ese centro profundo que supone la cultura auténtica.

Toda didáctica tiene un protocolo, al menos, aquel que intenta una solución a la cobertura. El protocolo es un esquema secuenciado y recurrente de elementos significantes previsto que concretan el azar del mundo. La cobertura tiene que ver con una nueva distribución de la cultura en contenidos, en conocimiento, esto obliga a tener una proporción gobernable y administrable entre los que enseñan y los que son enseñados. Como el trabajo del maestro se multiplica; la simplificación, dosificación, elección, facilitación, tiene que ver con esa multiplicación. La cultura, a la que un Estado ofrece acceso, a través de los sistemas educativos tiene para el joven Nietzsche, al menos, un problema. El problema de la educación que ofrece el Estado es el rasero, el nivel de esfuerzo al que está el acceso a una auténtica cultura. La autenticidad de esta cultura radica en su alta y estrecha medida. El rasero no puede ser el promedio, la cobertura no tiene que ser un promedio de cultura para todos. Los estudiantes tendrían que pasar por un trabajo de acceso ciego a la cultura, para que este acceso lleve a regiones privilegiadas, análogas a la excelencia griega, recontextualizada, sí, pero con gran cantidad de trabajo incorporado.

En las conferencias, el joven orador, cuenta una anécdota en la que se encuentran un maestro, un discípulo y unos intrusos espías, estudiantes desaforados, pero ya interesados en el saber (por eso se quedan a espiar). El maestro y el discípulo esperan por mucho tiempo a alguien y conversan mientras, sobre la educación. Los dos estudiantes desaforados habían estado practicando el tiro con ruidosas pistolas. Se encuentran los personajes y hay un regaño por parte del maestro, se separan. Luego, los estudiantes escuchan de manera furtiva un diálogo apasionado entre el maestro y el discípulo. Uno, de los que escuchan, es el orador. Posteriormente, los cuatro personajes se encuentran e intercambian palabras que resultan ser exposiciones sobre las instituciones educativas. Al final de la quinta conferencia aparece, a lo lejos, el personaje al que han estado esperando el maestro y su discípulo, estos han dado muestra de veneración en la espera. El sabio se acerca, envuelto en una inaudita compañía, jóvenes bulliciosos. Esta conducta pueril no se esperaría de semejante sabio, al cual se le atribuye cierto aborrecimiento por la juventud descarriada.
El joven Nietzsche usa la analogía para poner en discusión el lugar de la educación media en el camino que toma la cultura. Una de las oposiciones centrales, es aquella que se establece entre una idea de acceso a una cultura auténtica y otra que simplifica, divierte y hace ruido, como las voces de los jóvenes y sus pistolas. El conferencista pone a elegir, con la analogía, entre un legado cultural en la educación profunda y total y una simplificación de la cultura con la educación extensa y universal. El deseo por acceder a niveles escasos y costosos de la cultura moviliza una rivalidad voluntariosa consigo mismo; mientras que la distribución equitativa de la cultura no puede movilizar aquello que todos poseen por derecho: el mediano nivel operativo de las mayorías. Nietzsche arremete contra el periodismo y cierta filología. A la primera por superficial, a la segunda por alienada. Superficial como solución para la cobertura, para la máxima extensión de la comprensión inmediata, urgente y repentina, que es la opinión y la percepción. Alienada en la filología porque le es suficiente el estudio de la etimología en el legado de una cultura auténtica.

Para que una didáctica sea un objeto técnico tendría que haber una relación de causalidad recurrente entre pensamiento y vida. Una didáctica que se asocie a la vida, exclusivamente, así desayunen o no los estudiantes, es un aparato separado de la articulación social. Aquella didáctica que se asocia al pensamiento no es un objeto técnico, es un utensilio que define su linaje con la reproducción. Cada didáctica es una parte de las posibilidades, pensamiento y vida tienen que subsistir en la didáctica como objeto técnico. Simondon lo ve de esta manera,

El objeto técnico individualizado es un objeto que fue inventado, es decir producido, por un juego de causalidad recurrente entre vida y pensamiento en el hombre. EI objeto que solamente está asociado a la vida o al pensamiento no es objeto técnico sino utensilio o aparato. No existe consistencia interna, porque no existe medio asociado que instituya una causalidad recurrente. (Simondon, 2007, p. 81)

Pero, como se dijo, hay una posible didáctica que se asocia en un esquema de causalidad recurrente, ese esquema se mantiene en la relación con la vida y con el pensamiento. La causalidad recurrente opera en un medio asociado, más que en el conjunto técnico cerrado con el que cierta didáctica entra en relación. El medio asociado de la didáctica puede ser la cultura universal, con ella se leería lo local, el contexto, el conjunto. Una didáctica que se asocie a la vida, por separado, es la misma que se busca en la cobertura, la garantía de la vida ordenada de una población abastecida, por 
lo tanto, gobernada. Una didáctica que se asocie al pensamiento pierde el mundo donde se despliegan las relaciones. Pero habría que hacer algo radical con ese abastecimiento de la población o con su sublimación, superar la supervivencia, cultivar el legado, cuidar el pensamiento comprometido con la vida.

\section{Epílogo}

Los saltos (aquí son dos) en los esquemas técnicos se mantienen en la misma jerarquía porque los esquemas de operaciones, como unidad técnica, subsisten y comparten el mundo en paridad de realización. Los saltos en los esquemas técnicos van de la abstracción magnífica en la artificialidad, hasta la concretización en lo humano. El esquema técnico del preceptor privado medieval tiene un salto en sus esquemas técnicos, da paso al esquema técnico del preceptor público, así es como aparece en la obra Didáctica Magna de Jan Amos Comenius. La educación, con la didáctica, se vuelve sistema, se concretiza con un salto en los esquemas de la técnica educativa y se acopla como cobertura de un servicio de conocimiento, que no de saber, porque el saber no podría transportarse.

Volviendo a la pregunta inicial ¿Cuál es el lugar que ocupa esta didáctica en un linaje técnico, operando con una analogía donde la didáctica es análoga a un objeto técnico?

En la educación estamos en el mismo esquema técnico propuesto e implementado por Comenius en el siglo XVII, la didáctico como analogía de un objeto técnico tiene un salto al sistema educativo de las naciones, que todavía se verifica. La inclusión en el sistema de aparatos como las plataformas de las tecnologías de la información y la comunicación está previsto en el esquema técnico de la Didáctica Magna. Podría continuarse por esta vía con la pregunta por la asincronía de un manual como este, un manual habla también desde lo remoto con una estructura de búsqueda. La educación pública sigue teniendo el mismo esquema que privilegia el saber, dando un rodeo por la relación con el saber para llegar a lazo social, es a propósito del saber que se edifica un lazo educativo. Una didáctica aquí tendría un esquema técnico estructural, como se ha dicho, efecto de un salto en el linaje técnico. Es imposible administrar esta didáctica porque el método, la fórmula empírica (si la hubiera) estaría encaminada a disponer la selección de un legado saber, menos por el contenido que el tipo de relación. Entonces se contaría, en el linaje de este objeto técnico, con una analogía formal con la didáctica en tres esquemas técnicos, la didáctica del preceptor privado, el preceptor público y una posible didáctica en la formación. Esta última, entendida como la posibilidad de que la relación de saber tenga más relevancia que el contenido, pero que sea posible solo a condición del saber en la escuela.

En esta investigación habría tres esquemas técnicos, el de una didáctica privada renacentista (de la que no se trató), una didáctica pública de la cobertura (desde Didáctica Magna) y una posible didáctica formativa en propuesta. Entre estos esquemas técnicos se verifican unos saltos que es objeto preliminar en esta reflexión dentro de un proyecto de investigación. En fases posteriores de la investigación se podrá retomar la matriz técnica que se propone aquí para actualizarla, tomando la referencia empírica de un diseño que podrá ser contenido de otro texto. Sin embargo, para un trabajo empírico, se pudiera intentar medir las operaciones de los objetos técnicos, aceptados como tales y ensamblados en esquemas de operación distinguibles, por lo tanto, susceptibles de intervención. La reflexión contenida en este texto es independiente del contexto porque despliega unos conceptos que generalizan, en operaciones técnicas, los esquemas técnicos de un linaje de la didáctica, como si fuera un objeto técnico. Funciona así en cualquier contexto, aunque habría que verificar las excepciones. En la posible didáctica formativa no podría ser posible el autoaprendizaje, Comenius apoya esta idea. Como se ha dicho con la postura desplegada en el texto, es necesario un lazo social a expensas del saber, donde se aprende también a querer saber.

Finalmente, la analogía que se emplea en esta reflexión investigativa es distinta a la hermenéutica, en todo caso sería una heurística (Koen, 1985, p. 95). Pero no. La reflexión ha ido por otro camino porque va detrás de una ontogénesis del objeto técnico. Más que ser una interpretación que produzca un sentido, es la reconstrucción de los esquemas técnicos en que operan los objetos técnicos. Por su puesto que incluso esto produce un sentido, pero habría que ver cuál pudo haber sido este. En esta reflexión se delimita, con consideraciones estructurales, un objeto técnico: la didáctica en tanto esquema técnico de enseñanza en la educación. 


\section{Referencias}

Bernstein, B. (1990). La construcción social del discurso pedagógico. Textos seleccionados. Prodic-El Griot.

Bernstein, B. (1997). La estructura del discurso pedagógico, Clases, códigos y control. Ediciones Morata.

Bustamante, G. (2017). Carl Gustav Jung y la educación. Pedagogía y Saberes, 46, 65-75.

Charbonnier, G. (2006). Entrevistas con Claude Lévi-Strauss. Amorrortu Editores. (Original publicado en 1961).

Combes, M. (2017). Simondon, una filosofía de lo transindividual. Editorial Cactus.

Comenius, J. (2013). Didáctica magna. Ediciones Akal. (Original publicado en 1632).

Ende, M. (1985). La historia interminable. Editorial Alfaguara. (Original publicado en 1979).
Koen, B. (1985). El método de ingeniería. Universidad del Valle.

Nietzsche, F. (2010). Sobre el porvenir de nuestras escuelas. Tusquets Editores. (Original publicado en 1872).

Platón, (1961). Primer Alcibíades. Aguilar.

Simondon, G. (2007). El modo de existencia de los objetos técnicos. Prometeo libros. (Original publicado en 1958).

Simondon, G. (2009). La individuación, a la luz de las nociones de forma e información. Ediciones La Cebra y Ediciones Cactus. (Original publicado en 1969).

Simondon, G. (2017). Sobre la técnica. Cactus. (Original publicado en 1953-1983)

Sobel, D. (2006). Longitud. Editorial Anagrama.

Trujillo, A, (2017). Formación en máquina. [Tesis doctoral]. Universidad Pedagógica Nacional (UPN), Bogotá D.C. 